\title{
A Note on the Relationship between Relative Precipitation Frequency and Percent of Correct Forecasts
}

\author{
Richard J. Reed \\ Department of Atmospheric Sciences, University of Washington, Seattle, Wash. 98195
}

\begin{abstract}
Ramage (1982) has presented evidence, based on extensive verification data for precipitation forecasts, that the percent of correct forecasts decreases approximately linearly with increasing precipitation frequency. However, only a limited range of precipitation frequency was examined in his study. Here the theoretical reltionship between these quantities is derived for the full range of precipitation frequency with use of a model that assumes the forecasts to be unbiased and the Heidke Skill Score to be independent of precipitation frequency. It is shown that for this simple model the relationship is quadratic, the percent correct dropping from $100 \%$ for a relative precipitation frequency of zero to a minimum value for a relative frequency of 0.5 and rising again to $100 \%$ for a relative frequency of 1.0. The data presented by Ramage appear to fit the quadratic relationship well, within the limited range shown in Fig. 4 of his article.
\end{abstract}

In his recent contribution, "Have precipitation forecasts improved?", Ramage (1982) states two "firm impressions" gained from reading local forecast analyses: 1 ) forecast accuracy deteriorates as precipitation frequency increases and 2) winter forecasts are better than summer forecasts.

The purpose of this note is to point out that rule 1) applies only to regions for which relative precipitation frequency (PF) is less than 0.5. As Ramage has suggested, for those rarer regions for which relative precipitation frequency exceeds 0.5 , the rule is the reverse (provided the forecaster has the good sense to predict "precipitation" in doubtful situations rather than "no-precipitation"). This fact would have been obvious had Figs. 2-4 of Ramage's paper covered a greater range of precipitation frequencies and had the "noprecipitation forecast" lines in the figures been changed to "precipitation forecast" lines upon passing through $\mathrm{PF}=0.5$.

The reason for the behavior in question is well known and is often cited when arguing that percent correct $(\mathrm{PC})$ is a poor measure of forecast skill. In a climate where it never rains, the

\footnotetext{
${ }^{1}$ Authors should submit manuscripts for this section directly to Dr. Robert W. Burpee, Editor, Focus on Forecasting, Hurricane Research Division, Atlantic Oceanographic and Meteorological Laboratory, NOAA, Gables One Tower, 1320 S. Dixie Highway, Coral Gables, Fla. 33146. Three copies of each manuscript (text and illustrations), prepared in accordance with "Information for Contributors" on the inside covers of a recent issue of an AMS research journal, are required.
}

(c) 1983 American Meteorological Society forecaster scores $100 \%$, whether skilled or not, simply by always predicting no precipitation. In a climate where it rains infrequently, the unskilled forecaster will score below, but close to, $100 \%$. Similarly, in a hypothetical climate where it always or nearly always rains, the unskilled forecaster will score at or near $100 \%$ simply by always forecasting rain.

As a further point of interest, we inquired whether the true relationship between $P C$ and $P F$ is really a straight line relationship, as suggested by the linear fits shown in Ramage's article. A straight line obviously applies in the case of unskilled forecasts in which the forecaster always forecasts precipitation or never forecasts precipitation. But is it the likely relationship when the forecaster possesses skill?

An insight concerning this question is gained by considering the Heidke Skill Score, $S$, which is defined as the number of correct forecasts less the number correct due to chance divided by the total number of forecasts less the number correct due to chance (Brier and Allen, 1951). For unbiased forecasts this score takes the form:

$$
S=\frac{\mathrm{PC}-\left[(\mathrm{PF})^{2}+(1-\mathrm{PF})^{2}\right]}{1-\left[(\mathrm{PF})^{2}+(1-\mathrm{PF})^{2}\right]},
$$

where $\mathrm{PC}=$ the total percent of correct forecasts (expressed here as a fraction) and PF is the relative precipitation frequency. The term "unbiased" means that the forecaster predicts precipitation with the same frequency that it occurs, a simplifying assumption that is often more or less obeyed in practice. Rearrangement of Eq. (1) yields PC as a function of $S$ and PF.

$$
\mathrm{PC}=2(1-S)(\mathrm{PF})^{2}-2(1-S) \mathrm{PF}+1
$$

For the case of constant skill, that is, the case in which forecasts for different regions or time periods, characterized by different observed precipitation frequencies, exhibit the same skill $S$, it is seen from Eq. (2) that a quadratic relationship exists between $\mathrm{PC}$ and PF. This relationship is plotted in Fig. 1 for $S=0.5, S=0.25$ and for $S=0$. The latter represents the case in which the forecaster predicts precipitation and no-precipitation with the correct frequencies, but lacks skill in predicting individual events. Also shown is the linear relationship that applies for the case of biased forecasts in which either precipitation or no-precipitation is always forecast. 


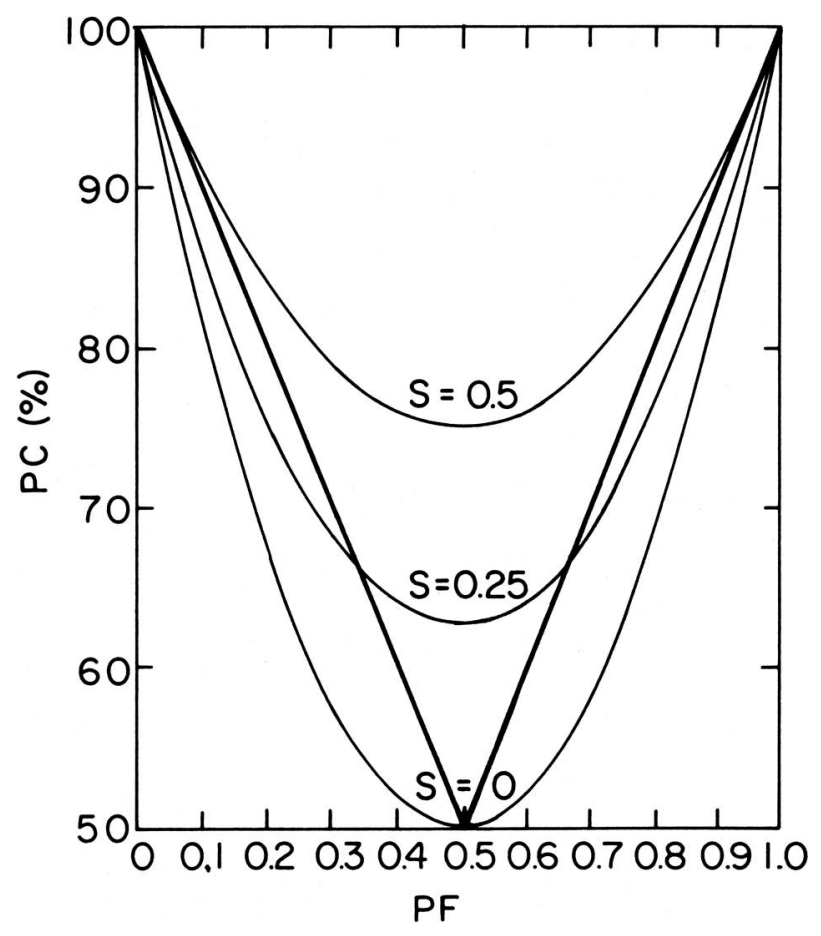

FIG. 1. Relationship between percent correct forecasts (PC) and relative precipitation frequency $(\mathrm{PF})$ for unbiased forecasts with uniform skill score $(S)$ for different values of the skill score.

It is remarkable that the curve for $S=0.5$, a value chosen for its simplification of Eq. (2) and without knowledge of the actual skill scores of the forecasts used in constructing Fig. 4 of Ramage's paper, yields a fit to the data that is almost surely superior to, or that easily could be adjusted to be superior to, the linear fit indicated in that figure. It would be interesting to know the skill scores connected with his data and whether the scores tend to cluster around the value 0.5 . Another point of interest is that it is better for the unskilled forecaster to always forecast precipitation if $\mathrm{PF}>0.5$ and no precipitation if $\mathrm{PF}<0.5$, rather than to vary the forecast from day to day in an unbiased way.

The forecaster with intermediate skill $(S=0.25)$ is in an ambiguous position. In climates for which $\mathrm{PF}<0.33$, the forecaster's best option is always to predict no precipitation and in climates for which $\mathrm{PF}>0.67$, always to predict precipitation. Only in climates with $0.33<\mathrm{PF}<0.67$ should the forecaster employ his forecasting skill. It is demonstrated easily that for skill scores between 0 and 0.5 the critical frequencies $\mathbf{P F}_{\mathrm{crt}}$, or intercepts of the quadratic and linear relationships, that determine which of the foregoing options should be employed are given by

$$
\begin{gathered}
\mathrm{PF}_{\mathrm{crt}}=\frac{1-2 S}{2-2 S} \text { for } 0<\mathrm{PF}<0.5 \\
\mathrm{PF}_{\mathrm{crt}}=\frac{1}{2(1-S)} \text { for } 0.5<\mathrm{PF}<1.0 .
\end{gathered}
$$

For $S \geq 0.5$, the forecaster should employ his or her skill whatever the precipitation frequency.

Although the foregoing considerations are mainly of academic interest, they should not be allowed to obscure the basic conclusion of this and Ramage's study: percent correct (PC) by itself is a virtually worthless measure of forecast skill.

Acknowledgment. This paper was supported by the National Science Foundation under Grant No. ATM81-03697. The paper is contribution No. 644, Department of Atmospheric Sciences, University of Washington.

\section{References}

Brier, G. W., and R. A. Allen, 1951: Verification of weather forecasts. In Compendium of Meteorology, AMS, Boston, pp. 841-848. Ramage, C. S., 1982: Have precipitation forecasts improved? Bull. Am. Meteorol. Soc., 63, 739-743.

announcements (continued from page 147)

\section{Study to improve industrial air pollution monitoring methods}

The U.S. Environmental Protection Agency (EPA) has awarded a \$545 128 contract to PEDCo Environmental, Inc. of Cincinnati, Ohio, to develop and evaluate improved methods for monitoring air pollutants from a variety of industrial sources.

William J. Mitchell of EPA's Environmental Monitoring Systems Laboratory at Research Triangle Park, N.C., project officer for the study, said the work will include both laboratory and field studies focusing on the chemical manufacturing, iron, and steel industries; refineries, and asphalt processing plants; and coal-fired power plants.

"Our goal is to develop improved methods for monitoring air pollutants from these large industrial sources, to determine the precision and accuracy of the methods, and to assure that the methods are used correctly," Mitchell said.

PEDCo will provide technical assistance in the development of monitoring equipment and techniques tailored for specific industrial sites. They will also assist EPA in developing and distributing standard samples of air pollutants for use in nationwide performance surveys of companies that conduct stack testing with EPA methods, and in calibration of monitoring equipment. 